\title{
BIBECHANA
}

A Multidisciplinary Journal of Science, Technology and Mathematics ISSN 2091-0762 (Print), 2382-5340 (Online)

Journal homepage: http://nepjol.info/index.php/BIBECHANA

Publisher: Research Council of Science and Technology, Biratnagar, Nepal

\section{Design and development of low-range frequency modulated signal (F.M.) transmitter}

\author{
Abhishek Shrivastava \\ Pradesh, India. \\ *Email: abhi6191995@ gmail.com \\ Article history: Received 20 September, 2017; Accepted 28 October, 2017 \\ DOI: http://dx.doi.org/10.3126/bibechana.v15i0.18279 \\ This work is licensed under the Creative Commons CC BY-NC License. \\ https://creativecommons.org/licenses/by-nc/4.0/

\section{(7) (5)}

Electronics and Communication Engineering, Jaypee Institute of Information Technology, NOIDA, Uttar

\section{Abstract}

Frequency Modulated (FM) signal transmitter is a small device that can transmit Frequency Modulated signal over short range. [1] This document consists of most simple and economical technique for building a FM transmitter using basic electronic components like resistor, capacitor, inductor etc. The FM transmitter receives human voice signals though microphone. It further amplifies it, modulate it over carrier and finally transmit it. Assuming favorable conditions, output of transmitter can be received by anyone who tunes it in frequency of our transmitter. Here, I have described Circuit diagram, its working, components required, uses of various components in our circuit, its practical applicability. The design is simulated using NI Multisim and is further implemented on bread-board. This design is capable of transmitting signal for distance of radius $20 \mathrm{~m}$, tuned at 97.1 MHz One could clearly hear sound produced at microphone of transmitter.

Keywords: Inductor; LC oscillator; Telescopic antenna; 2N3904 bipolar transistor.

\section{Introduction}

FM transmitter is an electronic device, which produces frequency-modulated waves with the help of an antenna. A transmitter generates FM waves for various purposes such as communication, broadcasting a message etc. Furthermore, FM signals are less prone to interference as compared to AM signals due to higher bandwidth. Also, it is less susceptible to noise [1,2]. The signal transmitted has a limited range for its reception, as we increase our distance from source, the signal received is merged with noise and further more noise component dominates signal transmitted and hence message cannot be received successfully after certain distance due to obstacles. The source of power is $9 \mathrm{v}$ dc battery, which starts discharging after constant power supply for around 6 hours [3]. The information that is provided to the transmitter is in the form of an electronic signal. This includes audio from a microphone. The transmitter combines the information signal that is to be carried with the RF signal (the carrier). This is called modulation. In an FM transmitter, the information is added to the radio signal by slightly varying the radio signal's frequency.

\section{Materials \& Methods}

The components used in our design are easy to find and easy to implement. The circuit designing and implementing part can also be done using breadboard but as it is not suitable high frequency circuits. Further Components are shown in Table 1. 
Table 1:

\begin{tabular}{|c|c|c|c|}
\hline Part Name & Quantity & Value & Description \\
\hline M1 & 1 & $12-3 \mathrm{db}$ & Microphone \\
\hline R1 & 1 & $470 \Omega$ & Resistor \\
\hline R2 & 1 & $4.7 \mathrm{k} \Omega$ & Resistor \\
\hline C1 & 1 & $4.7 \mathrm{pF}$ & Capacitor \\
\hline C2 & 1 & $20 \mathrm{nF}$ & Capacitor \\
\hline C3 & 1 & $50 \mathrm{pF}$ & Capacitor \\
\hline C4 & 1 & $0.01 \mathrm{uF}$ & Capacitor \\
\hline C5 & 1 & $0.1 \mathrm{uF}$ & Capacitor \\
\hline L1 & 1 & $0.05 \mathrm{uH}$ & Inductor \\
\hline Q1 & 1 & $2 \mathrm{~N} 3904$ & General Purpose Transistor \\
\hline V1 & 1 & $9 \mathrm{~V}$ & DC power Supply \\
\hline
\end{tabular}

Microphone: Microphone is a transducer, which converts sound energy to electric energy. The microphone used in our design is 'Electret Condenser microphone'[4] It consists of parallel plate capacitor which has one plate as fixed and other plate being movable. The movable plate is called diaphragm. When sound strikes diaphragm it starts moving, thus in turn changing capacitance of capacitor, which in turn results in flow of variable current.

Transistor (2N3904): Transistor 2N3904 is a medium gain general-purpose transistor. It is widely used for low power amplification. It has transition frequency of around $300 \mathrm{MHz}$ with a minimum current gain of 100. The Circuit Diagram is shown in Figure 1, which was stimulated in NI Multisim 10.

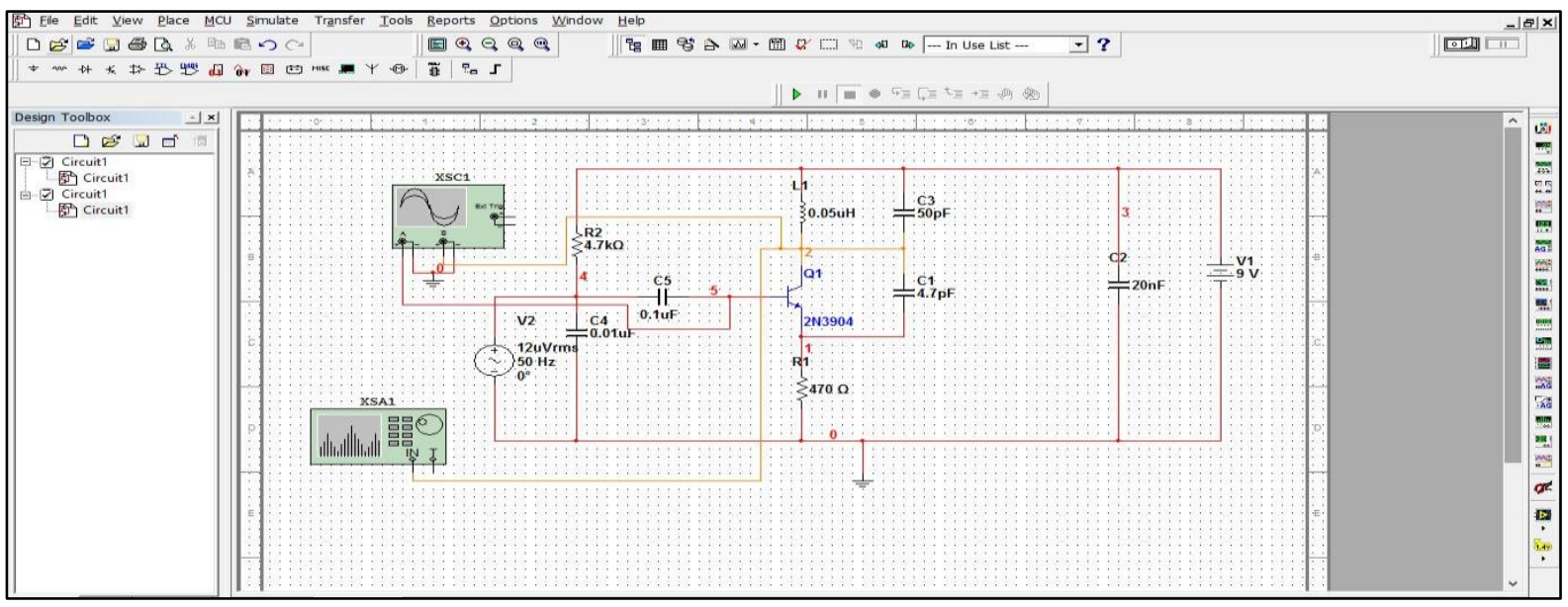

Fig.1: Circuit diagram.

\section{Discription of Circuit}

The circuit basically operates in 4 steps. Firstly a condenser microphone takes input, then amplifier does amplification, the amplified signal is modulated with frequency being generated by LC oscillator and finally antenna transmits the signal. The inductor L1 and capacitor C 3 forms an oscillating tank circuit along with the transistor 2N3904. As long as the current exists across the inductor coil L1 and the capacitor $\mathrm{C} 3$, the tank circuit will oscillate at the resonant carrier frequency for FM modulation. Whereas Capacitor $\mathrm{C} 1$ acts as a negative feedback to the oscillating tank circuit. The modulated signal from the antenna is radiated as radio waves at FM frequency band. Antenna is nothing but a simple copper wire of $20 \mathrm{~cm}$ or more long. 


\section{Input Stage:}

Inside the microphone, a capacitive sensor diaphragm is present. It vibrates according to the air pressure changes and generates AC signals. The capacitor C4 and C5 can be thought of as a frequency-dependent resistor (called reactance). The Capacitor C5 separates microphone from transistor. Speech consists of different frequencies and the capacitor impedes them. The net effect is that $\mathrm{C} 4$ modulates the current going into the transistor. Using a large value for $\mathrm{C} 4$ reinforces bass (low frequencies) while smaller values boost treble (high frequencies). The input stage of Multisim circuit is shown in Figure 2. Here we used an A.C. voltage source instead of microphone for simulation purpose.

\section{Amplification Stage:}

The sound signal converted by microphone to alternating signal has very low power, hence if we modulate it directly over the carrier and transmit it, it would not be possible to demodulate it and retrieve the original signal from it. The resistance R1 and R2 are used to bias the BJT, it is being illustrated in Figure 3.

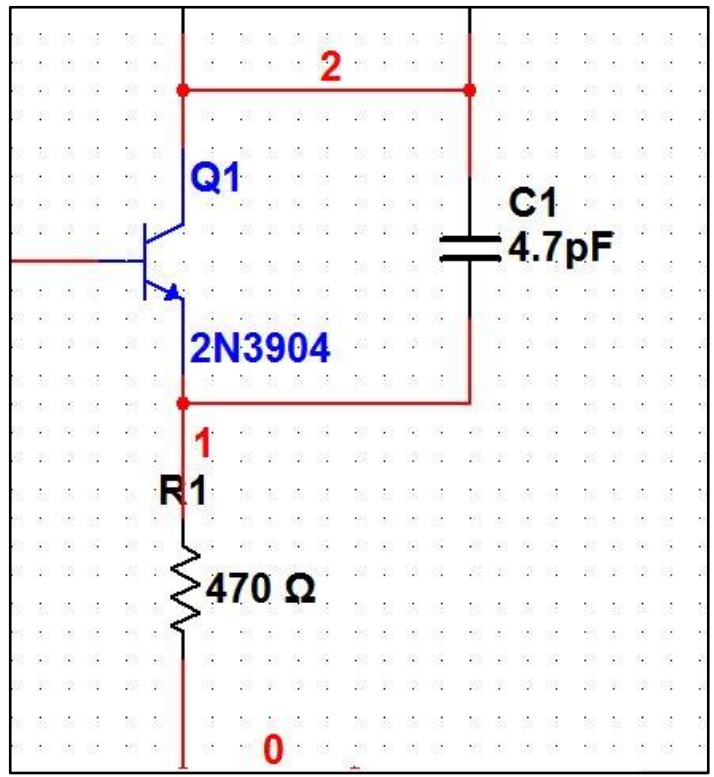

Fig.2: Input Stage.

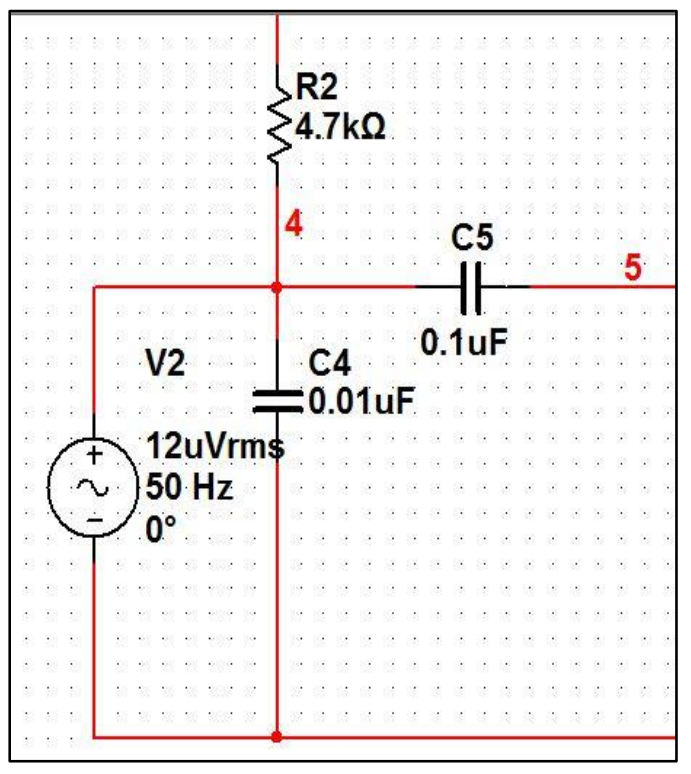

Fig. 3: Amplification Stage.

Tank Circuit: The Inductor and capacitor together form an oscillating tank circuit or a resonant circuit oscillating at frequency given by,

$$
\mathrm{f}=\frac{1}{\sqrt{2 * \pi * L * C}}
$$

where, L \& C are values of Inductor and Capacitor forming tank circuit respectively. Here L1 and C3 forms an oscillating tank circuit with frequency $=97.1 \mathrm{MHz}$. It is further displayed in Figure 4.

\section{Final Transmission of FM signal}

Now, the signal after amplification by BJT is further modulated with carrier signal generated by tank circuit. After successful modulation the wave is transmitted using Antenna. It is displayed in Figure 5. 


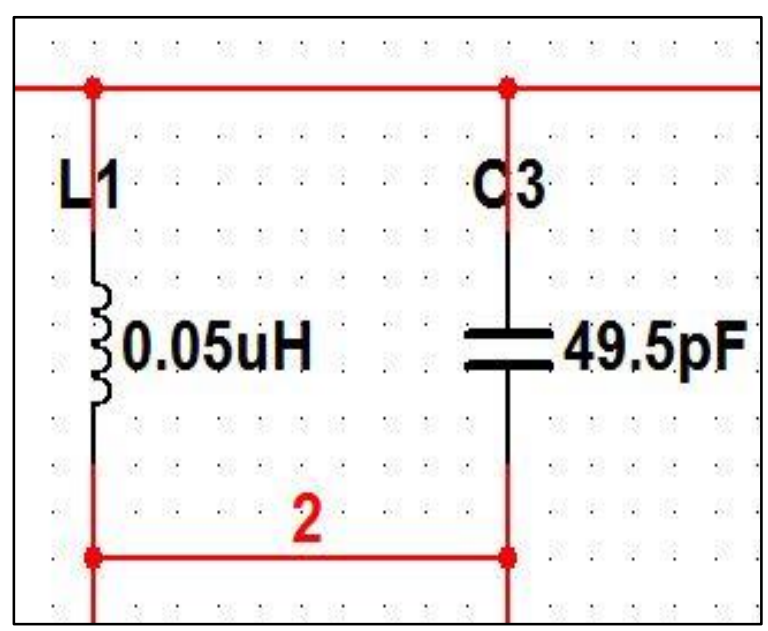

Fig. 4: Tank Circuit.

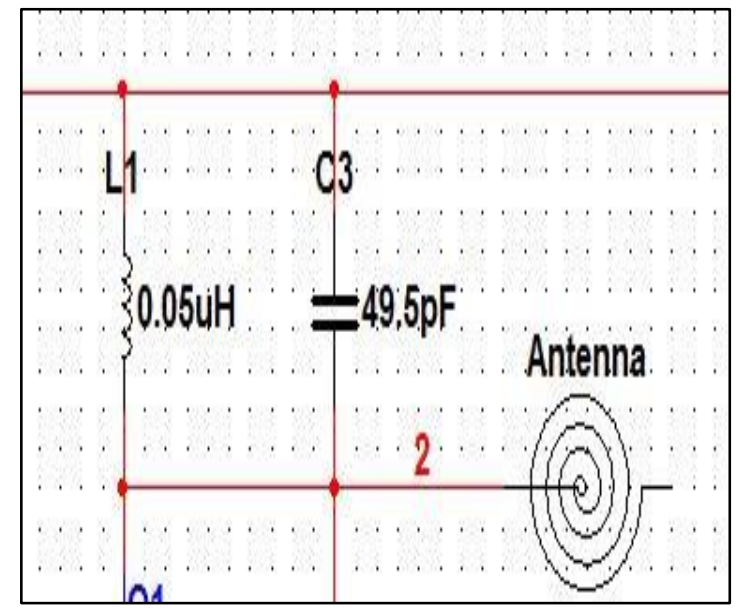

Fig. 5: Transmission using Antenna.

\section{Results and Discussion}

\section{Simulation using NI Multisim 10}

FM transmitter can be simulated using tools from Multisim. This circuit is simulated using NI Multisim 10. Firstly, circuit is created using Multisim, using the tools and parts from library, dedicated for circuit designing. The main purpose of using Multisim to simulate the design is to affirm that values of inductor capacitor, etc. are under the bounds. After the circuit is designed it is further simulated and analyzed using Spectrum Analyzer and virtual oscilloscope present in Multisim. Also, applying probes at antenna shows the values of various parameters at output of circuit. The results of simulation are illustrated in Figure 6.

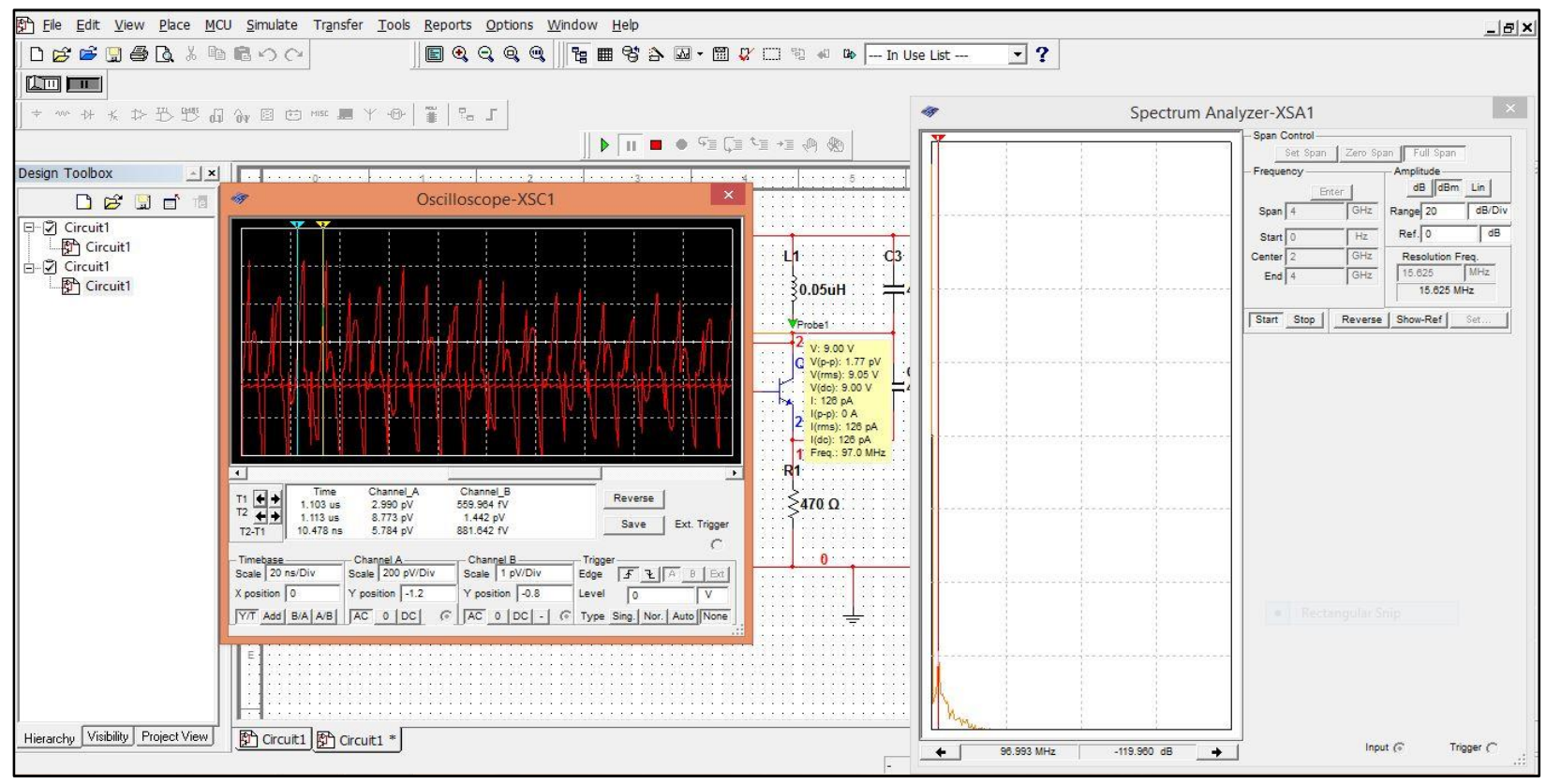

Fig. 6: Results of Simulation. 


\section{Interpretation by Oscilloscope}

As it can be seen in Figure 6, the oscilloscope displays the output waveform of FM signal that is transmitted through antenna. The output wave can be clearly seen through oscilloscope. As our design is very basic, the effect of noise can be seen in output as well. Clearly it can be noted that the output waveform has time period of $10.4 \mathrm{~ns}$ which is approx. $97 \mathrm{MHz}$. Slight variations are observed due to effect of noise.

\section{Interpretation by Spectrum Analyzer}

The spectrum of output waveform can be analyzed using Spectrum Analyzer. It can be clearly observed from the graph of spectrum analyzer of Figure 6, the peak of spectrum (bandwidth) is at $96.663 \mathrm{MHz}$, approximately $97 \mathrm{MHz}$. Also the effect of noise can be observed as the spectrum is not idle as it is expected to be of a sinusoidal wave.

\section{Interpretation by Probe Marker}

Using the Probe marker placed at output during simulation, the frequency of output signal can be clearly seen from Figure 6 as 97.0 MHz., along with various other parameters such as current, voltage etc.

\section{Implementation and Testing}

\section{Implementation}

Further after all the simulation results are achieved as per desired, we further implement circuit designed on breadboard first. All the components listed in Table-1 are further arranged and assembled in circuit as per design requirement of our FM transmitter. Implemented circuit on bread board is shown in Figure-7, along with battery source.

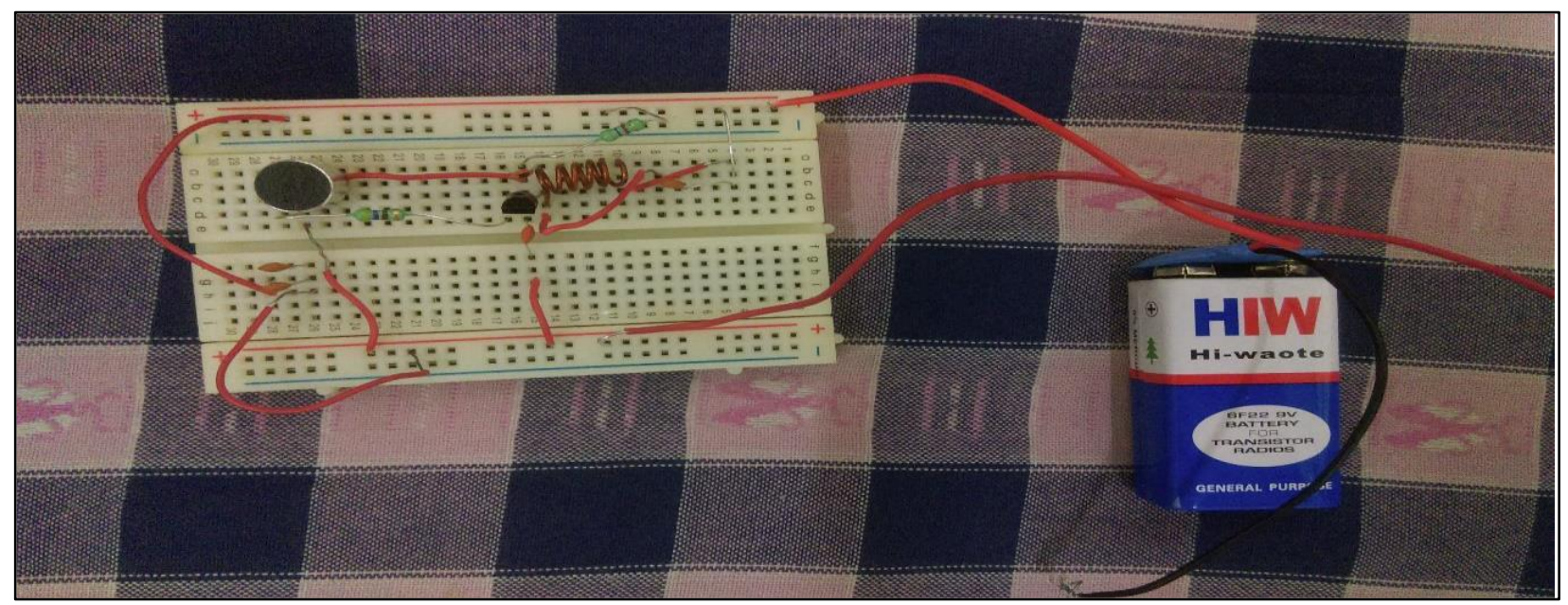

Fig. 7: Practical implementation.

\section{Testing}

The implemented circuit is further tested. To test the transmitter, the tank circuit is tuned properly so that frequency generated can be easily modulated with message signal. Now, Both FM transmitter and receiver are switched on. The receiver is tuned to $97.1 \mathrm{MHz}$ and voice signal is being transmitted. Instantly the voice signal is heard clearly at the receiver. Furthermore, to check the range of transmitter, a constant voice signal is applied at input and receiver is moved away from the transmitter slowly. As distance increased, the impact of noise started dominating our message signal. Also, as our transmitter is producing a low power signal, output can be obtained clearly in a radius of $20 \mathrm{~m}$ around 
transmitter. The Figure 8 shows output of our design being verified using Digital Storage Oscilloscope (DSO).

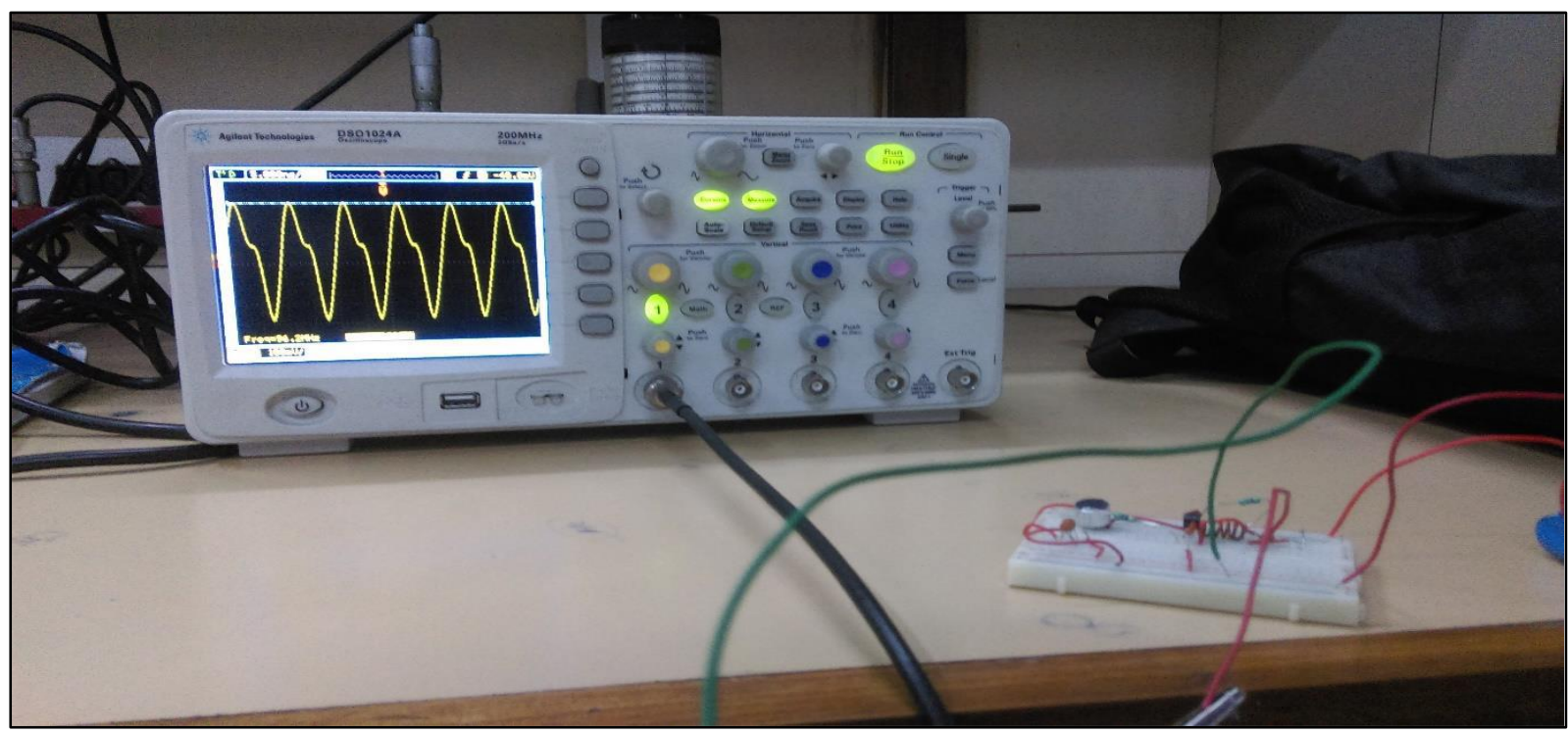

Fig. 8(a)

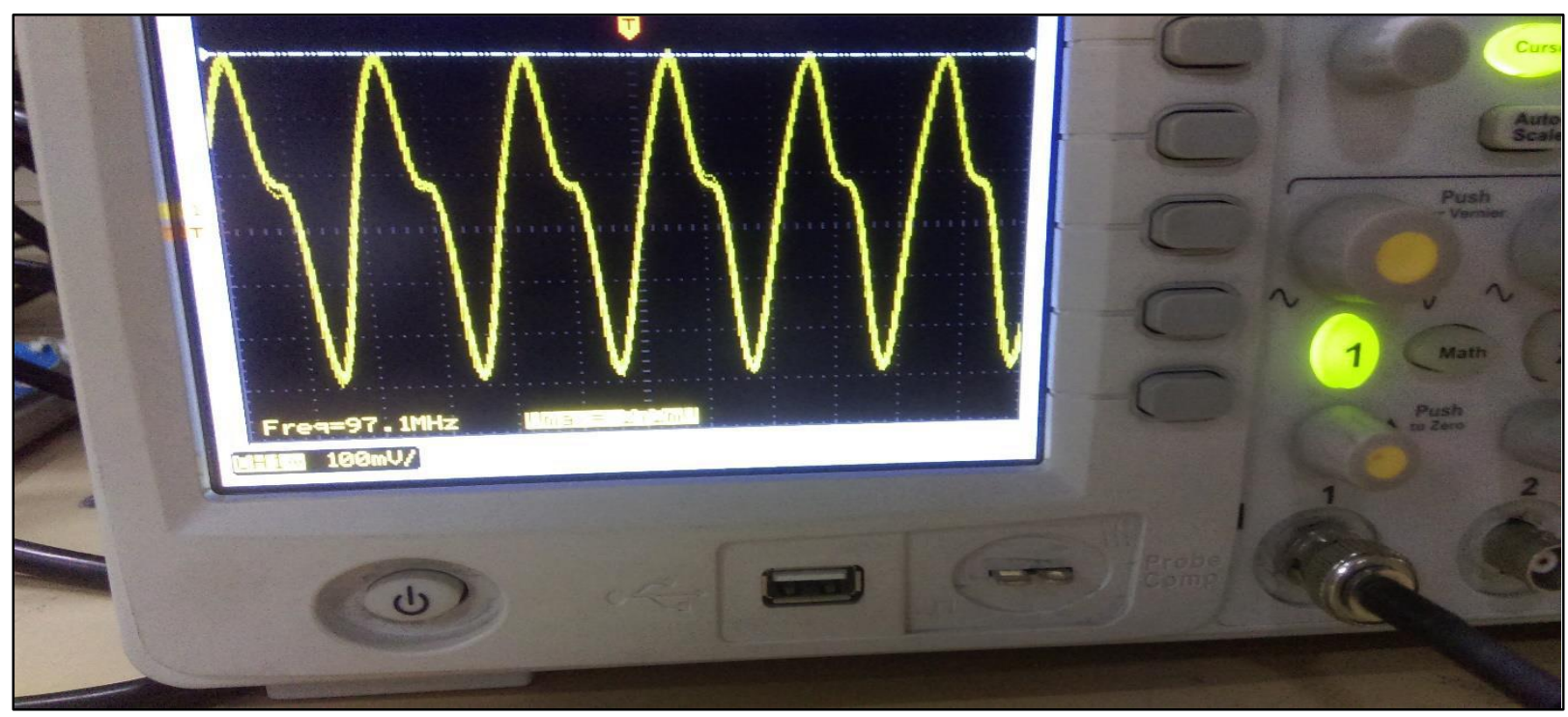

Fig. 8(b)

\section{Conclusion}

The human voice transmitted, was received at output on $97.1 \mathrm{MHz}$ frequency, provided conditions are favorable for wireless transmission. For extending the range and power of FM transmitter, one can apply another level of Amplification after Second stage, that would in turn further amplify the signal and then it can be transmitted, the more the power of signal transmitted, greater is its range and more noise immune it becomes. Also, to improve efficiency one may check the voltage of source applied 
and assure it is $9 \mathrm{~V}$ for above circuit. Furthermore, one should implement the design on PCB board as bread-board is not preferred for high frequency circuits.

\section{References}

[1] R.L. Boylestad, Introductory Circuit Analysis, Eleventh Edition, Prentice Hall, 2007.

[2] N. Braga, Pirate Radio and Video: Experimental Transmitter Projects, Newnes Butterworth-Heinemann Publishing, 2001..

[3] B.P. Lathi., Modern Analog and Digital Communication Systems, Third edition, Oxford University Press. 1998.

[4] T. L. Floyd, Principles of Electric Circuits, Eighth Edition, Upper Saddle River, NJ: Prentice Hall, 2007. 